\title{
THz instrumentation for the Herschel Space Observatory's heterodyne instrument for far infrared
}

John C. Pearson, Imran Mehdi, John S. Ward, Frank W. Maiwald, Robert R. Ferber, et al.

John C. Pearson, Imran Mehdi, John S. Ward, Frank W. Maiwald, Robert R. Ferber, Henry G. LeDuc, Erich T. Schlecht, John J. Gill, William A. Hatch, Jonathan H. Kawamura, Jeffrey A. Stern, Todd C. Gaier, Lorene A. Samoska, Sander Weinreb, Bruce Bumble, David M. Pukala, Hamid H. Javadi, Bradley P. Finamore, Robert H. Lin, Robert J. Dengler, James R. Velebir, Edward M. Luong, Raymond Tsang, Alejandro Peralta, Mary Wells, William Chun, Jonas Zmuidzinas, Alexandre Karpov, Thomas Phillips, David Miller, Alain E. Maestrini, Neal Erickson, Gerald Swift, K. T. Liao, Michael Paquette, "THz instrumentation for the Herschel Space Observatory's heterodyne instrument for far infrared," Proc. SPIE 5498, Millimeter and Submillimeter Detectors for Astronomy II, (8 October 2004); doi: 10.1117/12.552397

Event: SPIE Astronomical Telescopes + Instrumentation, 2004, Glasgow, United Kingdom 


\title{
THz Instrumentation for the Herschel Space Observatory's Heterodyne Instrument for Far Infrared
}

\author{
J. C. Pearson ${ }^{a}$, I. Mehdi ${ }^{a}$, J. S. Ward ${ }^{a}$, F. Maiwald ${ }^{a}$, R. R. Ferber ${ }^{a}$, H. G. Leduc ${ }^{a}$, E. T. Schlecht ${ }^{a}$, J.

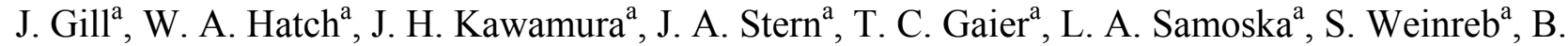 \\ Bumble $^{a}$, D. M. Pukala ${ }^{a}$, H. H. Javadia, B. P. Finamore ${ }^{a}$, R. H. Lin ${ }^{a}$, R. J. Dengler ${ }^{a}$, J. R. Velebir ${ }^{a}$, E. \\ M. Luong ${ }^{\mathrm{a}}$, R. Tsang ${ }^{\mathrm{a}}$, A. Peralta ${ }^{\mathrm{a}}, \mathrm{M}$. Wells ${ }^{\mathrm{a}}$, W. Chun ${ }^{\mathrm{a}}$, J. Zmuidzinas ${ }^{\mathrm{b}}$, A. Karpov ${ }^{\mathrm{b}}$, T. G.

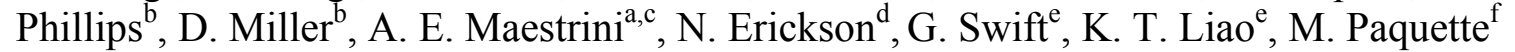 \\ a Jet Propulsion Laboratory, California Institute of Technology, Mail Stop 301-429, 4800 Oak Grove \\ Dr., Pasadena CA 91109 (USA); \\ ${ }^{\mathrm{b}}$ California Institute of Technology, Mail Stop 320-47, Pasadena, CA 91125 (USA); \\ cLISIF - Université Paris VI, 4, place Jussieu - case 25275252 Paris cedex 05 (France); \\ ${ }^{\mathrm{d} U n i v e r s i t y ~ o f ~ M a s s a c h u s e t t s / A m h e r s t, ~ A m h e r s t, ~ M A ~} 01035$ (USA); \\ ${ }^{\mathrm{e}}$ Northrop Grumman Corp, One Space Park, Redondo Beach, CA 90278 (USA); \\ ${ }^{\mathrm{f}}$ Millitech LLC, 29 Corporate Dr East, Northampton, MA 01060 (USA)
}

\begin{abstract}
The Heterodyne Instrument for Far Infrared (HIFI) on ESA's Herschel Space Observatory utilizes a variety of novel RF components in its five SIS receiver channels covering 480- $1250 \mathrm{GHz}$ and two HEB receiver channels covering 1410$1910 \mathrm{GHz}$. The local oscillator unit will be passively cooled while the focal plane unit is cooled by superfluid helium and cold helium vapors. HIFI employs W-band GaAs amplifiers, InP HEMT low noise IF amplifiers, fixed tuned broadband planar diode multipliers, high power W-band Isolators, and novel material systems in the SIS mixers. The National Aeronautics and Space Administration through the Jet Propulsion Laboratory is managing the development of the highest frequency (1119-1250 GHz) SIS mixers, the local oscillators for the three highest frequency receivers as well as W-band power amplifiers, high power W-band isolators, varactor diode devices for all high frequency multipliers and InP HEMT components for all the receiver channels intermediate frequency amplifiers. The NASA developed components represent a significant advancement in the available performance. This paper presents an update of the performance and the current state of development.
\end{abstract}

Keywords: THz Receivers, Local Oscillators, GaAs Amplifiers, Multiplied Sources

\section{INTRODICTION}

A fully modern heterodyne receiver designed for autonomous operations, such as the Heterodyne Instrument for Far Infrared (HIFI) ${ }^{1}$ scheduled for launch on the European Space Agency's Herschel Space Observatory, requires a number of technological improvements for implementation. In HIFI, the NASA funded "High Frequency Subsystem" has played a major role in developing and providing enabling technology for this demanding flight application. The fundamental HIFI instrument application is an observatory with unparalleled capability and flexibility. The requirements include nearly complete frequency coverage from 480 to $1910 \mathrm{GHz}$. This paper updates a previous paper ${ }^{2}$ in the performance and the current state of development. In many cases, the final flight hardware has been flight qualified, built and accepted. Two other papers in this conference deal with local oscillators ${ }^{3}$ and $1.2 \mathrm{THz}$ SIS mixers ${ }^{4}$ in more detail. A final paper ${ }^{5}$ in this conference discusses the overall HIFI local oscillator subsystem architecture and development.

*Send correspondence to John.C.Pearson@jpl.nasa.gov; phone 1-818-354-6822; fax 1-818-393-2430 
The HIFI instrument is a seven-channel, single-pixel, dual polarization, double sideband receiver system with one receiver operated at time. Two separate spectrometers analyze both E- and H-Plane polarizations. The wide band spectrometer is a $4 \mathrm{GHz}$ bandwidth array acousto-optical spectrometers ${ }^{6,7}$. The high resolution spectrometer is narrower banded and uses an autocorrelation spectrometer ${ }^{8,9}$. The HIFI receivers, which are built around superconductor-insulator-superconductor (SIS) technology will employ a 4 to $8 \mathrm{GHz}$ IF. The channels employing phonon cooled hot electron bolometers (HEB) will employ a 2.4 to $4.8 \mathrm{GHz}$ IF and an IF conversion unit to fold these IF bands into the spectrometer bands. Table 1 provides a frequency table of the HIFI receiver bands and denotes the mixer and local oscillator bands.

Table 1 Frequency bands of HIFI

\begin{tabular}{|l|l|l|l|l|l|l|}
\hline & Frequency & E-Mixer & H-Mixer & IF & LO \#1 & LO \#2 \\
\hline Band 1 & $480-641$ & SIS & SIS & 4 to 8 & $488-546$ & $560-633$ \\
\hline Band 2 & $639-801$ & SIS & SIS & 4 to 8 & $647-710$ & $724-793$ \\
\hline Band 3 & $799-961$ & SIS & SIS & 4 to 8 & $807-848$ & $862-953$ \\
\hline Band 4 & $959-1121$ & SIS & SIS & 4 to 8 & $967-1042$ & $1056-1113$ \\
\hline Band 5 & $1084-1274$ & SIS & SIS & 4 to 8 & $\mathbf{1 0 9 2 - 1 2 4 2}$ & $\mathbf{1 1 0 4 - 1 2 6 6}$ \\
\hline Band 6L & $1410-1704$ & NbN HEB & NbN HEB & 2.4 to 4.8 & $\mathbf{1 4 1 4 - 1 5 4 5}$ & $\mathbf{1 5 5 1 - 1 6 9 6}$ \\
\hline Band 6H & $1702-1910$ & NbN HEB & NbN HEB & 2.4 to 4.8 & $\mathbf{1 7 0 2 - 1 8 0 2}$ & $\mathbf{1 8 0 8 - 1 9 0 5}$ \\
\hline
\end{tabular}

Bold $=$ NASA/JPL Supplied Subsystem

Italics $=$ NASA/JPL Supplied Components in subsystem

\section{SCIENCE REQUIREMENTS}

The HIFI instrument is designed as an observatory and must support a wide range of science investigations from the community at large. Approximately $2 / 3$ of the available observing time will be competed in open proposals. The core science planned for HIFI includes investigations of star formation, stellar lifecycles, the ISM, galaxies, and solar system objects. As a result, HIFI requires the best sensitivity possible, which translates into high optical throughput, cold optics and the lowest possible mixer and intermediate frequency amplifier noise temperatures. In the lower bands, the emphasis is on the water frequencies between $480 \mathrm{GHz}$ and $980 \mathrm{GHz}$. In the higher bands, HIFI opens the complete window above $980 \mathrm{GHz}$ where observations are nearly impossible from the ground. The velocity distribution in a spatially unresolved galaxy requires an instantaneous IF bandwidth of approximately $4 \mathrm{GHz}$, as does the desire to make comprehensive spectral surveys of objects using relatively small amounts of observing time. Lastly, the red shifts of strong lines in distant objects must be compensated for as well as separation of upper and lower sideband components, requiring the local oscillator to have a small frequency step size and nearly complete frequency coverage.

Limitations in local oscillator power-bandwidth at high frequencies require the mixers to operate with low levels of local oscillator power. The combination of sensitivity, bandwidth and limited local oscillator power leads to the use of cryogenic SIS or HEB mixers in conjunction with diplexer local oscillator injection. The need for ultimate sensitivity and stored cryogens require the IF amplifiers to have very low noise and very low power dissipation. For observatory operation, the local oscillator must have full frequency coverage and no mechanical tuning elements. At the time of the HIFI proposal, none of these requirements had been fully demonstrated in the necessary combination and significant development, especially in the local oscillator, was required. In this paper, we show how these requirements have been achieved and hardware developed for the flight instrument.

\section{MIXERS}

In a previous paper, ${ }^{2}$ we discussed the development of the SIS technology required for the HIFI band 5, covering 1184 to $1274 \mathrm{GHz}$. Since that time, the major tasks have been the engineering of the flight system and the resolution of a number of engineering details. These included improvement of the mixer magnet design to provide at least 1200 Gauss at the mixer with a maximum of $15 \mathrm{~mA}$ drive current. This was achieved along with a reduction of the total weight to less than 75 grams while providing sufficient robustness to survive a vacuum bake and 60 total thermal cycles to the operational temperature. The mixer unit is a modular design with a magnetic assembly, a main body, an optics 
assembly and the cover with its fasteners. The magnet assembly includes the circuit board, connector, back EMF resistor, deflux heater, coil and pole pieces. The main body includes the alignment references, the bias electronics, the IF matching network, the IF connector and the bias connector. The optics assembly is designed for replacement in the selection of devices and includes the mixer device, the lens and a retaining ring. The cover is assembled from 100 micron thick copper foil and is gold plated. Figure 1 shows an exploded view of the band 5 SIS mixer.

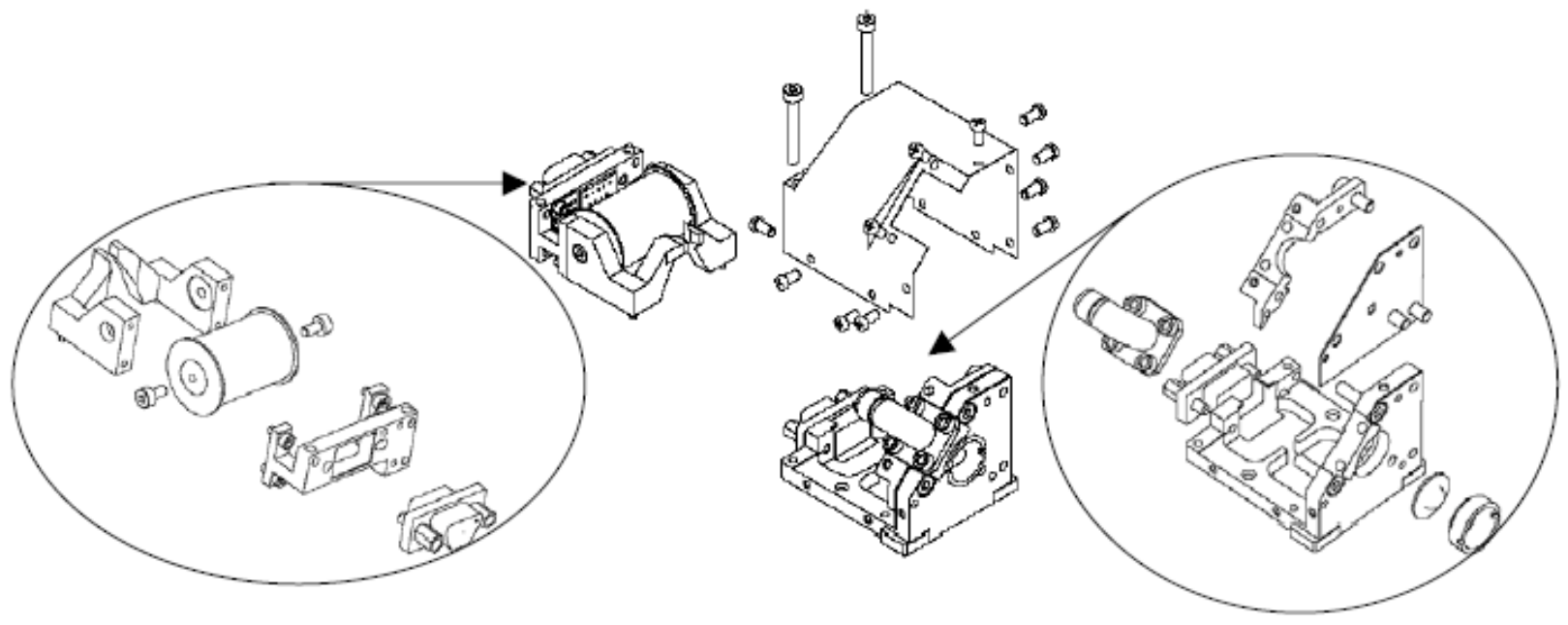

Figure 1: Assembly view of the HIFI band 5 SIS mixer. The optics assembly is shown on the right side of the bottom subassembly drawing.

The HIFI mixers are required to survive an involved ground test campaign, including as many as 20 thermal cycles in test and characterization prior to launch of the Herschel Spacecraft. Additionally, the mixer must survive acceptance vibration at the supplier, in the focal plane unit and finally in the acoustic test of the protoflight Herschel spacecraft before launch. Since Herschel uses stored cryogens, the mixers are launched cold and the protoflight tests are performed on a cold mixer unit. As a result, HIFI is performing mixer qualification vibration at LN2 temperature or colder if more convenient. The mixers are also vacuum-baked in the Herschel cryostat after each time the cryostat is opened to assure a sufficiently good vacuum for the launch hold. The combination of ground test environments and spacecraft environments combine to make a challenging qualification program.

The HIFI mixers have a two-stage qualification program. In the first stage, the suitability of all the parts materials and processes were evaluated relative to the spacecraft and test environments. Parts, materials and processes found not to survive reliably were changed and the test process repeated. The second stage is the formal qualification of the overall mixer to a plan addressing the specific environments in the HIFI test campaign and the Herschel launch environment. Since the mixers will see multiple acceptance tests, the acceptance vibration test at the supplier is a room temperature workmanship test. Suppliers will try to minimize the number of thermal cycles on the flight mixers to avoid undue stress.

The implementation of the SIS tunnel junction in band $5^{10,11}$ is with an epi-Nb ground plane, a thin $40 \mathrm{kA} / \mathrm{cm}$ AlN barrier directly on top of the ground plane followed by a counter electrode of NbTiN results in a very short junction height. This coupled with the 0.24 square-micron SIS junction size, results in a very high field needed to produce one quantum of flux in the device. The magnet in the band 5 mixer is constructed from a high purity $99.99 \%$ iron core, which is gold plated. The bobbin is then coated with parylene except for the ends, which mate to the pole pieces. The bobbin is wound with approximately 60,000 turns or 6000 feet of 25 micron diameter wire which is composed of a Niobium Titanium core, a copper cladding and formvar insulation. The result is a coil with a room temperature resistance of approximately $100 \mathrm{~K}$ ohms, that is superconducting with a critical current of about $1 \mathrm{amp}$. This magnet is to be operated with less than $15 \mathrm{~mA}$ of current. 
The twin-slot double tunnel junction design requires the simultaneous suppression of the critical-current (Ic) in both junctions. For a rectangular junction, the critical-current vs magnetic field curve has a sharp minima, so any nonuniformity in the manufacturing will make simultaneous suppression of Ic in both junctions difficult. As a result, the junctions are implemented in a diamond shape. The diamond shaped junction has a flatter minima of the critical current, so it is much easier to simultaneously suppress Ic in both junctions. The field is oriented perpendicular to the long dimension of the junction to minimize the field needed. Figure 2 shows a typical measured IF power vs magnetic field at the nominal bias point for this mixer configuration. The result is a mixer that is highly insensitive to variation in magnetic field and has a wide range where Josephson effects are adequately suppressed. Figure 3a-c show the SIS circuit and lithography quality of the band 5 SIS devices at progressively higher magnification. These devices have been successfully qualified for all the HIFI environments and have proven to be quite robust outside of the expected class 1 electrostatic discharge sensitivity due to the small junction size.

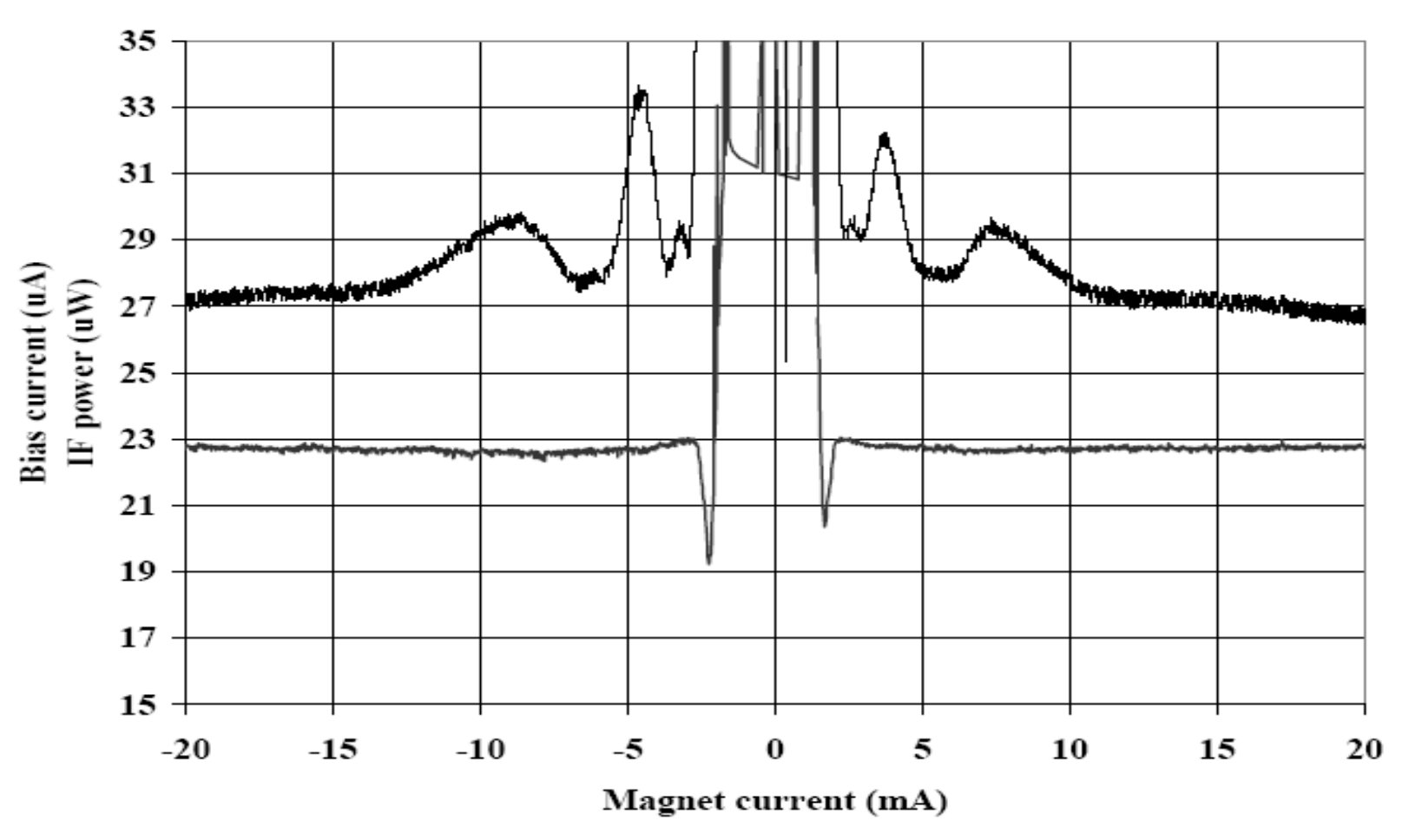

Figure 2. Band 5 mixer IF power and bias current at the nominal $2 \mathrm{mV}$ bias point with the 60,000 turn magnet coil for diamond shaped junctions.

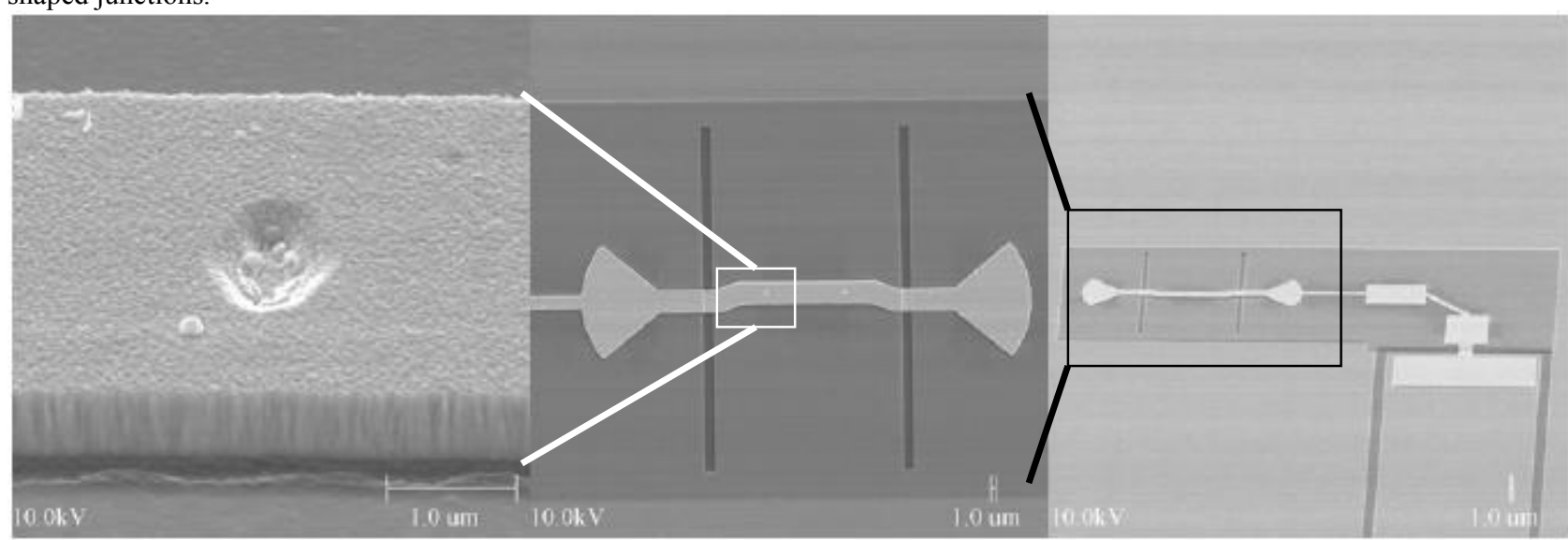

Figure $3 \mathrm{a}, 3 \mathrm{~b}$ and $3 \mathrm{c}: 3 \mathrm{a}$ the sub micron impression of the device as seen from the top metal. $3 \mathrm{~b}$ the twin slot antenna and the twin junction circuit (inverted relative to $3 \mathrm{c}$ ), $3 \mathrm{c}$ the SIS circuit plus the on chip IF matching network 
The hot electron bolometer (HEB) mixers for band 6 do not require a magnet, so the mass is less of a concern in the configuration. As a result, it was possible to machine the entire mixer out of aluminum panels and not resort to a thin cover. Figure 4 shows the assembly of the band 6 mixer. Two separate papers in this session discuss the HEB device and its performance in detail ${ }^{12,13}$. In the band 6 mixer, the DC and RF parts of the circuit are implemented on separate circuit boards. The construction and qualification approach is identical to the band 5 mixer with the same parts and processes being used whenever possible. Figure 5 shows the QM band 5 and QM band 6 mixers.

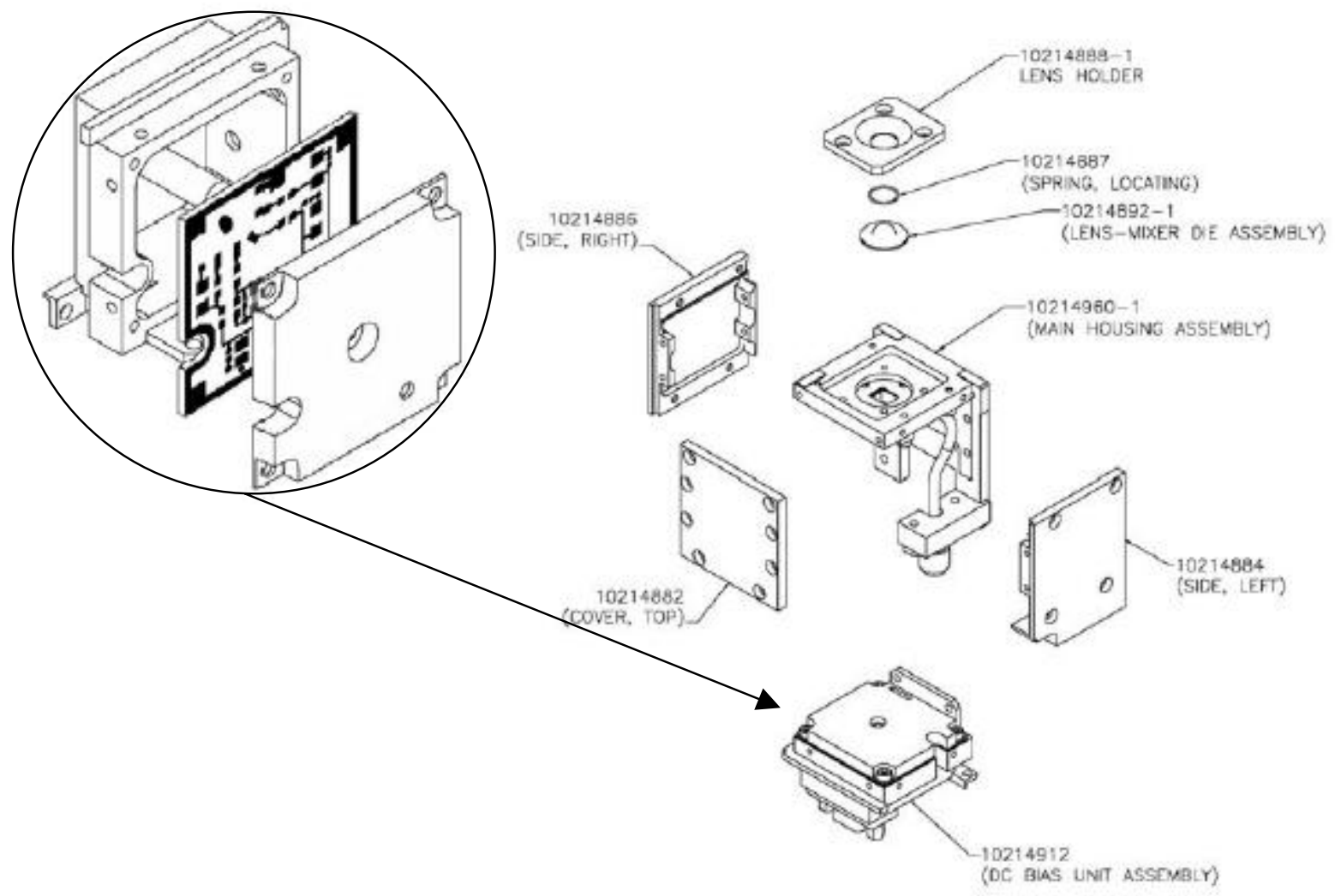

Figure 4: Assembly view of the HIFI band 6 mixer. The DC assembly is shown in the inset.

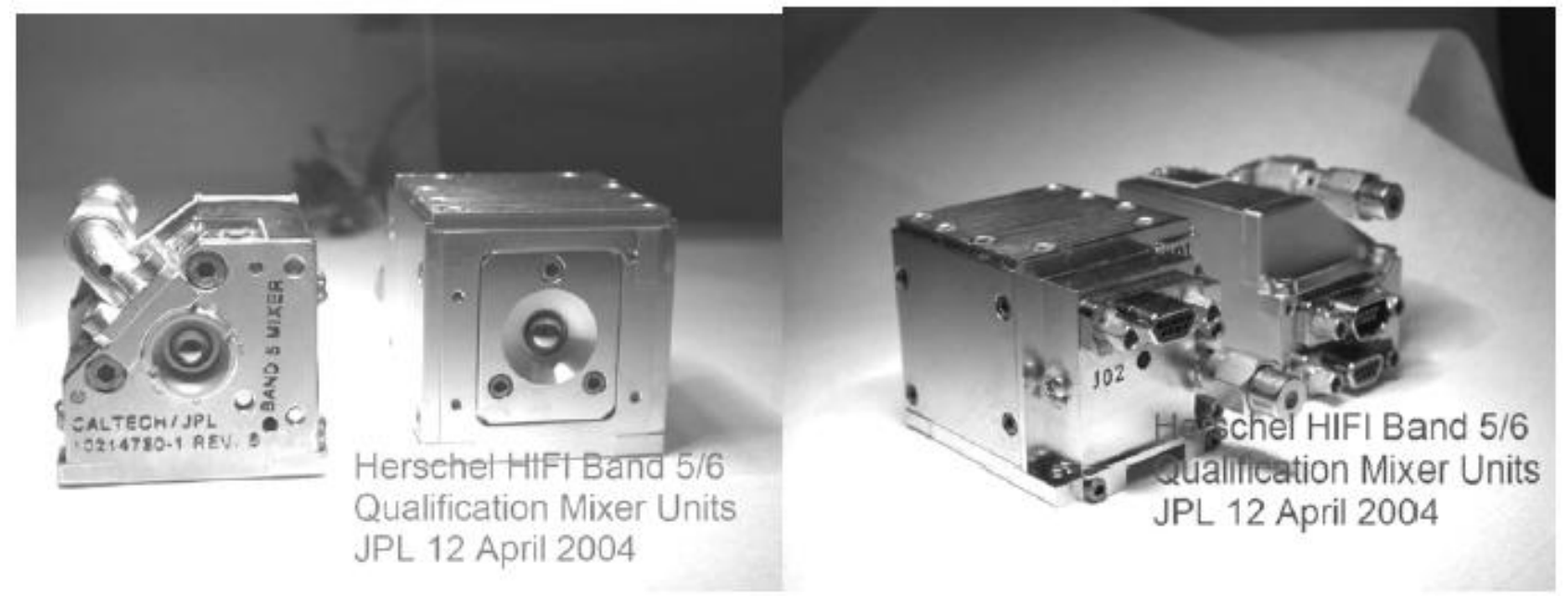

Figure 5: Qualification model band 5 and band 6 mixers 


\section{LOCAL OSCILLATORS}

The local oscillator unit for HIFI required new technology and implementation to achieve the broad bandwidth required. Due to the added complexity of cryogenic mechanisms, it is highly desirable to implement the local oscillator with no mechanical parts. As a result, traditional Gunn oscillator technology was quickly ruled out for HIFI. The HIFI local oscillator system uses a low frequency synthesizer, where tuning bandwidth is much easier to achieve, and multiplies the signal up to $\mathrm{THz}$ frequencies. The detailed architecture has been discussed previously ${ }^{14}$ and is updated in a separate paper in this volume ${ }^{5}$. A major task for the high frequency subsystem was to develop a W-band amplification stage $\mathrm{e}^{15}$ for HIFI.

The flight program consisted of the development and fabrication of nine engineering model units in five bands, 71-79.5 $\mathrm{GHz}, 80-92 \mathrm{GHz}, 88-99.5 \mathrm{GHz}, 92-106 \mathrm{GHz}$ and $100-112 \mathrm{GHz}$. 23 flight units were then built in the lower four of the bands. Of the engineering units, one was used in the qualification of the band 5 local oscillator chain, one was used to qualify the amplifier design, four were provided to the HIFI LO development model program, two were provided to the HIFI qualification model program and one was used as an evaluation unit for the flight MMICs. The flight models include two 71-79.5 GHz units, five $80-92 \mathrm{GHz}$ units, five $88-99.5 \mathrm{GHz}$ units and eleven $92-106 \mathrm{GHz}$ units. A qualification and test program was employed, based on Mil-Std-883, but modified for the cryogenic test and evaluation environment. The flight amplifiers are about 102 grams each and are just less than $6 \mathrm{~cm}$ long. A 21 pin MDM connector allows for two sets of gate and drain controls, gate and drain sense lines, as well as a return sense line. High current pins on the drain lines are paralleled. Figure 6 shows the schematic layout and the picture of the flight package.

The flight power amplifiers have all been constructed and they behave largely as expected. The amplifiers are operated in saturation to suppress the AM noise, which double side band receivers are sensitive to. As result, the drain voltage for the last stage of amplification effectively determines the output power. The result is that adjusting a single voltage can control the output power over at least $15 \mathrm{~dB}$. In order to verify that the amplifier was saturated and the power could be adjusted appropriately, a series of tests were performed. These included measuring $\mathrm{P}_{\text {out }} \mathrm{vs} \mathrm{P}_{\text {in }}$ as a function of frequency to check the gain and $\mathrm{P}_{\text {out }}$ vs the output stage drain voltage as a function of frequency. Figure $7 \mathrm{a}$ and $7 \mathrm{~b}$ shows a typical $\mathrm{P}_{\text {out }}$ vs $\mathrm{P}_{\text {in }}$ plot for an $80-92 \mathrm{GHz}$ amplifier and a typical $\mathrm{P}_{\text {out }}$ vs output frequency.
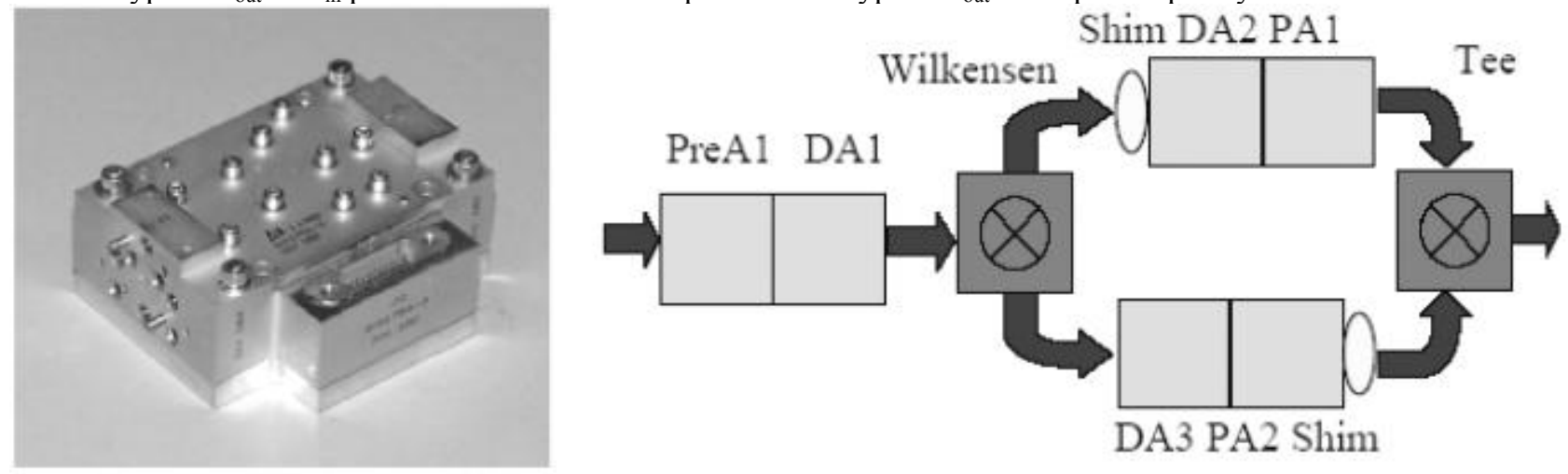

Figure 6: The schematic layout of the power amplifier and the engineering model amplifier package. The "PreA" is a low power preamplifier MMIC, the "DA\#" is a medium power driver amplifier MMIC, the "PA\#" is a high power amplifier MMIC and the "T" is a waveguide magic $\mathrm{T}$.

A second major consideration in achieving a wide bandwidth local oscillator is a good match between the high frequency components. In HIFI, the amplifier is driven by a varactor tripler and it drives a varactor doubler. In spite of the fact that the amplifier is implemented in a 90 degree hybrid, additional isolation was proven to help the overall multiplier chain performance. The problem was that no available isolator had sufficient power handling and low loss over enough bandwidth. The lowest loss design was the wide band design ${ }^{16}$ of Millitech, but this could not handle the RF power in vacuum and would not perform as desired below $80 \mathrm{GHz}$. As a result, two HIFI specific designs were developed. These utilized a carbon aluminum body to minimize coefficient of thermal expansion in the active area and a silicon disk to remove the heat generated by the RF power. The lower frequency design covers $\sim 65 \mathrm{GHz}$ to $92 \mathrm{GHz}$ and the high frequency design covers 85 to $110 \mathrm{GHz}$ in a $19 \mathrm{~g}$ and $16.5 \mathrm{~g}$ package. Figure 8 shows the isolator assembly. 
Typical isolation is $24 \mathrm{~dB}$ and typical insertion loss is $0.6 \mathrm{~dB}$ at $120 \mathrm{~K}$. The units were qualified for $200 \mathrm{~mW}$ dissipated internally. Millitech has delivered all 54 of the flight isolators for Herschel HIFI.

SN510@120K Pin vs Pout

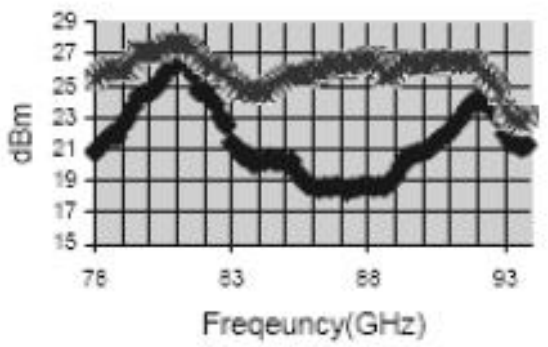

SN510@120K
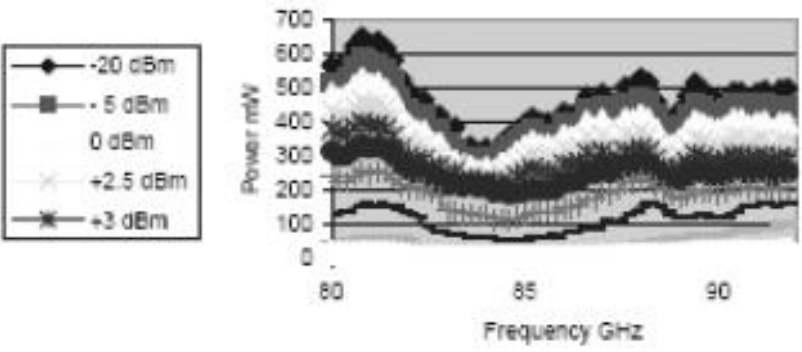

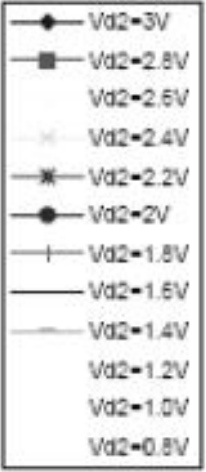

Figure $7 \mathrm{a}$ and $7 \mathrm{~b} .7 \mathrm{a}$ is the output power in $\mathrm{dBm}$ vs input power in $\mathrm{dBm}$ as a function of frequency for unit 506 at the operational temperature. $7 \mathrm{~b}$ is the $\mathrm{RF}$ output power in $\mathrm{dBm}$ vs the output amplifier drain voltage.

The big technical challenge in HIFI is achieving the required local oscillator power and bandwidth. The critical step in achieving this goal was the development of two planar diode processes to allow for balanced multipliers with on-chip tuning structures to be fabricated with the precision of e-beam lithography if necessary. The low frequency process ${ }^{17}$, which can be used up to approximately $1 \mathrm{THz}$, uses stepper lithography and requires mechanical thinning of the wafer to the desired thickness, typically 40,25 or 12 microns. The high frequency process ${ }^{17}$, which is intended for greater than $1 \mathrm{THz}$, uses e-beam lithography and an etch stop layer at 3 microns. The devices are chemically etched to the final 3 -micron thickness. Both processes support on-chip capacitors and beam leads. Figure 9 shows the types of devices that can be produced by the processes. A total of 4 diode production lots have been fully qualified and delivered to the HIFI instrument, completing the HIFI development.

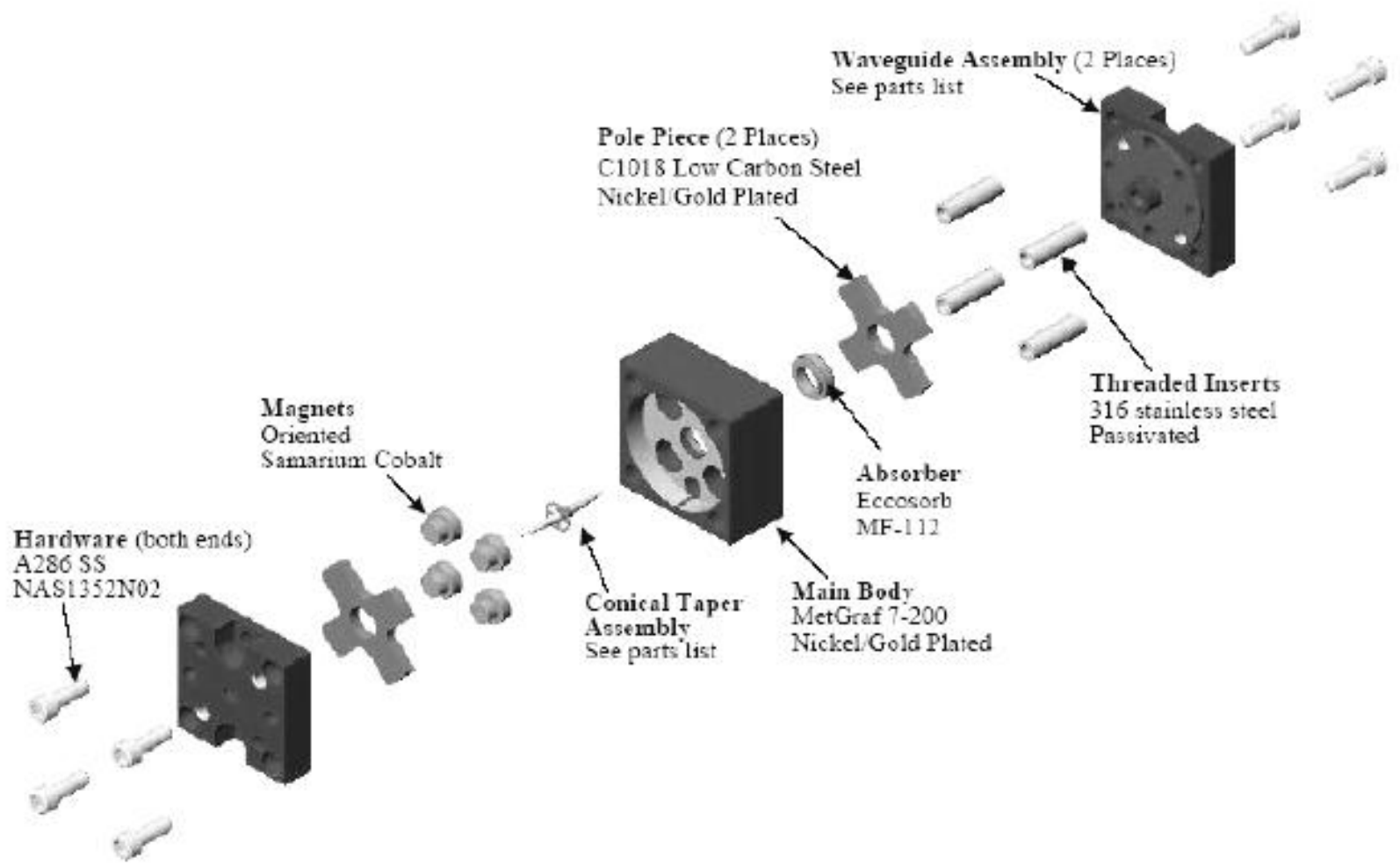

Figure 8: Assembly view of the HIFI W-band isolators showing the components. The conical taper assembly has an input taper, a silicon disk, a ferrite rod and an output taper. 
Implementing local oscillator chains that are wide banded and have a smooth bias characteristic, suitable for interpolation of bias points between measurement, requires careful optimization of the individual multiplier stages and careful selection of stages in the cascade to the full chain. If everything goes well, a chain can be built with significant bandwidth and sufficient output power. The HIFI band 5 local oscillator chains were originally required to cover 1127$1178 \mathrm{GHz}$ and 1192-1242 GHz respectively. The goal was to cover the full band with one chain and a prototype achieving this was demonstrated ${ }^{18}$. The major challenge of the flight hardware was the mechanically challenging alignment requirements for placement of the feed horn. A structure was designed with an adjustable flexure and a number of waveguide shims were developed to go in the $\mathrm{W}$-band path to adjust the length. This structure was successfully qualified for vibration and the thermal test environment of HIFI. The flight multiplier alignment achieves an accuracy of a few microns in the lateral displacement of the feed horn and a few tens of micron accuracy along the optical propagation axis. Figure 10 shows the two band 5 flight multipliers during the final alignment.

The performance of these multipliers achieves all the goals set forth in the HIFI program. They achieve the desired output power of 45 microwatts over the full band. The chain covering the required 1127-1178 GHz also covers more than $30 \mathrm{GHz}$ below the required band allowing HIFI to have redundancy on the key $1097 \mathrm{GHz}$ and $1113 \mathrm{GHz}$ water lines. The chain covering the required 1192-1242 also covers up to $1266 \mathrm{GHz}$, allowing HIFI to have an extra $24 \mathrm{GHz}$ of coverage.
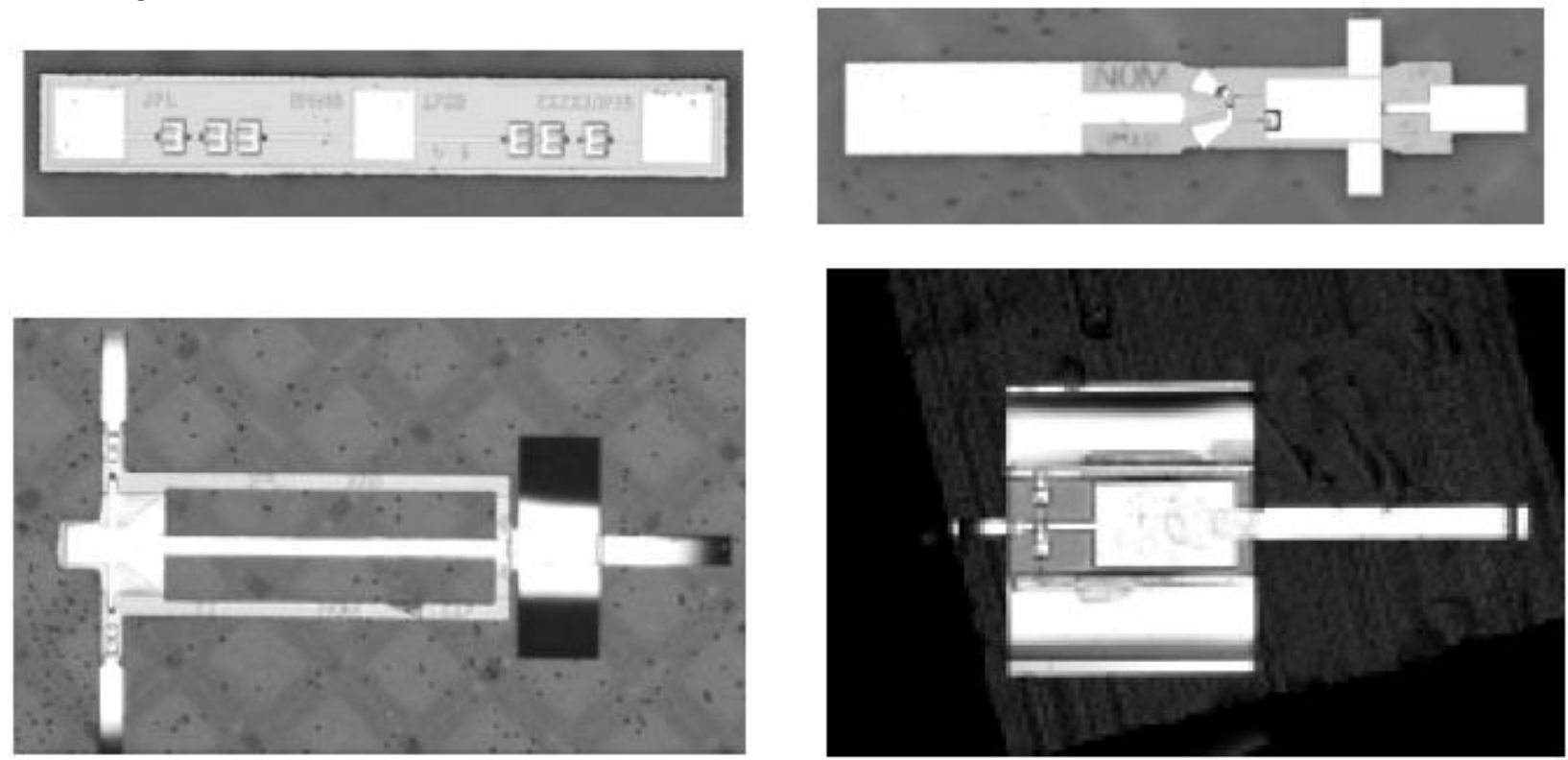

Figure 9a-9d. 9a top left, low frequency discrete doublers (no capacitor or beam leads), 9b top right, low frequency tripler (capacitor and beam leads), 9c bottom left, low frequency air dielectric or "substrateless" doubler 9d bottom right, high frequency or membrane tripler.

The last task for the high frequency subsystem to complete is the multipliers for frequencies between 1.41 and 1.91 THz. In this case, the state of the art is being pushed for machining of multiplier housings, fabrication of multiplier diodes and mechanical assembly. Mechanical tolerances on the final stages have to be on the order of 2 microns or less in critical areas. For frequencies below $1700 \mathrm{GHz}$, a x16 approach is planned, using a cascade of four frequency doublers starting from 88 to $106 \mathrm{GHz}$ in two chains. For frequencies above $1700 \mathrm{GHz}$, a x18 approach is planned, using a doubler followed by two triplers starting from 95 to $106 \mathrm{GHz}$ in two chains. The use of four stages, or the cascade of two triplers, complicates the construction of the high order multiplier chains. Both cases lead to increased standing wave problems between the multipliers and generally result in more power slope in the pass band. Figure 11 shows a $1.5 \mathrm{THz}$ and a $1.8 \mathrm{THz}$ chain prior to mounting on the adjustable structure with the power amplifier and isolators shown in Figure 10. The $1.8 \mathrm{THz}$ chain differs from the chain in Figure 10 by the cut out around the feed horn, which allows the smaller feed horn to be implemented in a large enough mechanical block to accommodate the 
necessary screws and alignment pins. Details on the construction of the 1.5 and $1.8 \mathrm{THz}$ multiplier chains have been presented previously ${ }^{19,20}$.

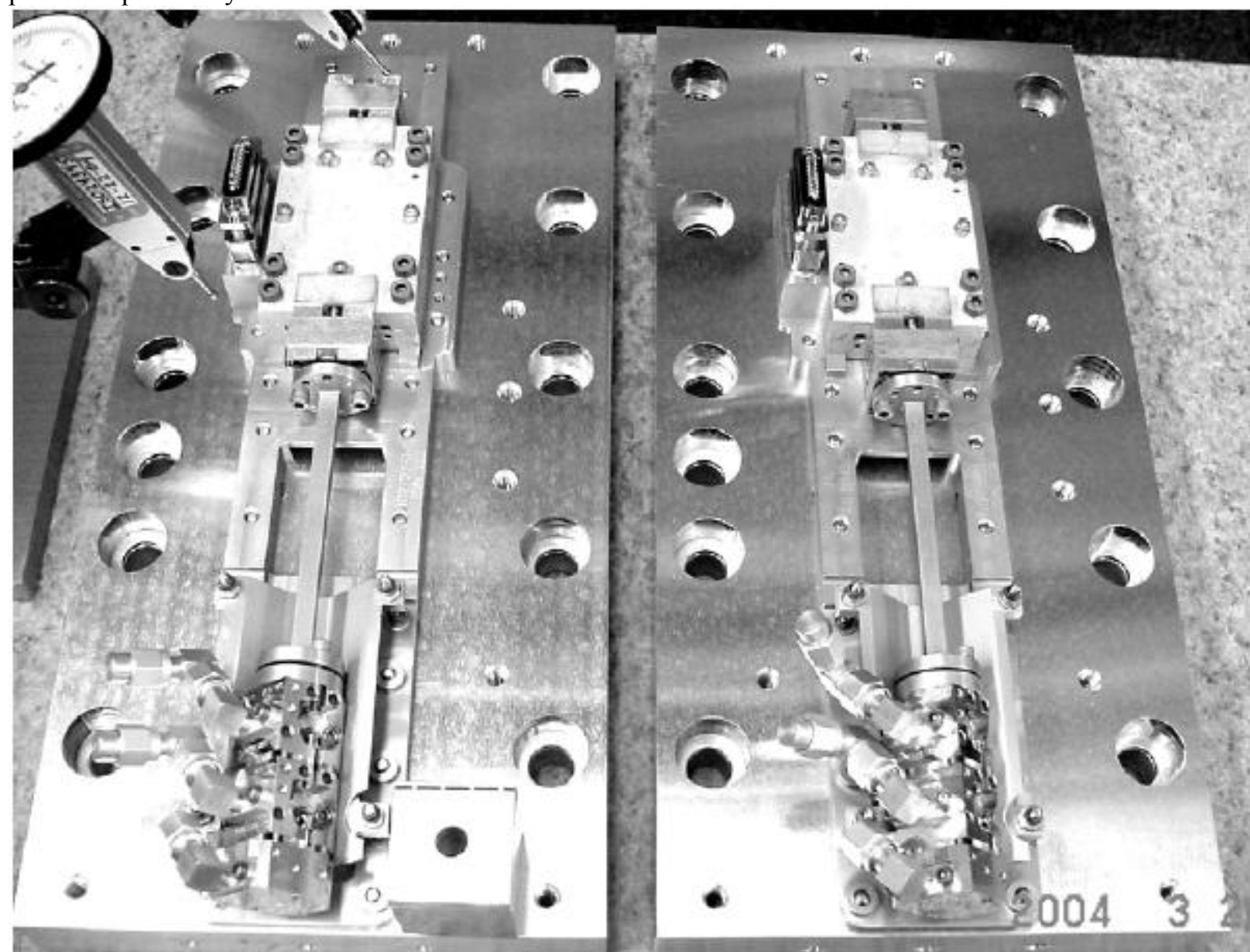

Figure 10. HIFI band 5 flight model multipliers in final alignment. From top to bottom there are an isolator, a power amplifier, an isolator a waveguide, a shim, a $200 \mathrm{GHz}$ doubler, a $400 \mathrm{GHz}$ doubler and a $1200 \mathrm{GHz}$ tripler with integrated feed horn.

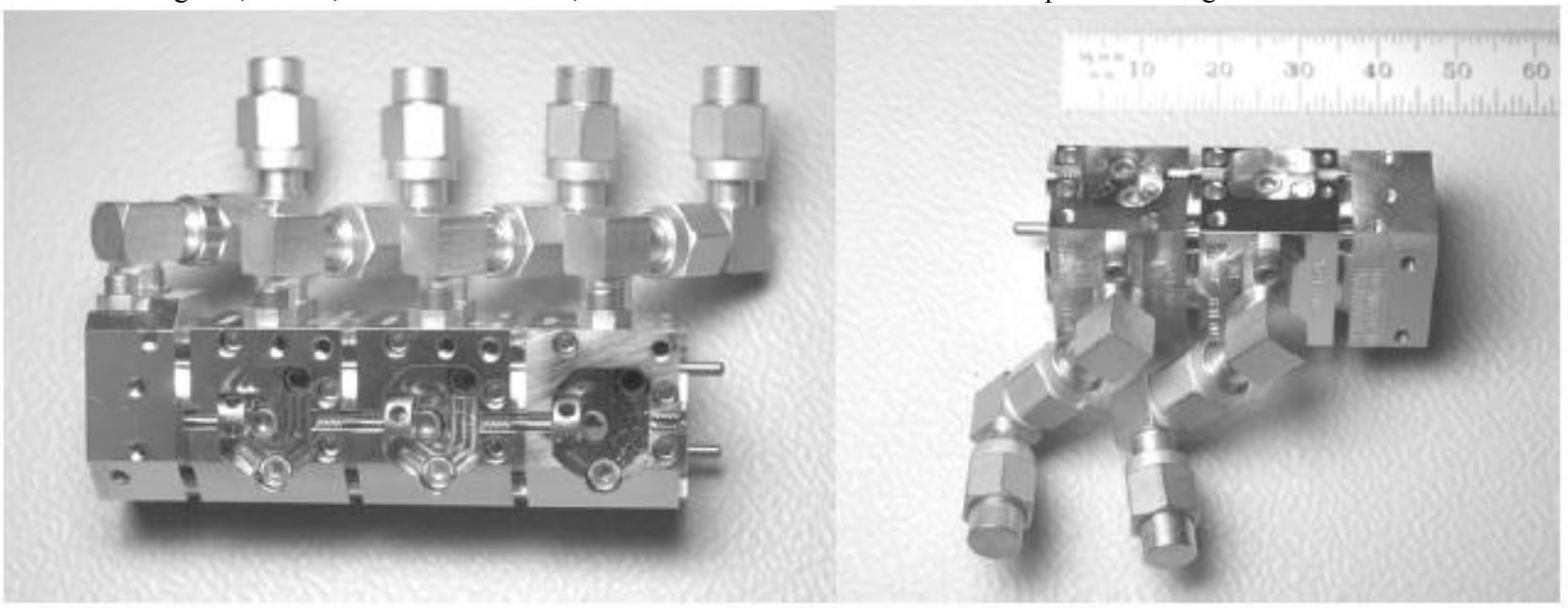

Figure 11. A four stage x16 multiplier consisting of four cascaded doublers and a three stage multiplier for $1.8 \mathrm{THz}$ consisting of a doubler and two triplers. It can be see that all multipliers with a waveguide input and output have the same exterior dimensions, as do all the final stage multipliers. 
Two years ago, the first $1.5 \mathrm{THz}$ results were reported ${ }^{2}$. Since then an enormous amount of progress has been made in realizing the HIFI band 6 multipliers. Currently the chain for $1.5 \mathrm{THz}$ has been well demonstrated and the individual flight stages constructed. All multiplier stages except the third stage are completely satisfactory and even that one is probably good enough. In the 1.7 to $1.9 \mathrm{THz}$ band, two different chains composed entirely of flight components have been assembled and tested. The chain covering 1.7 to $1.83 \mathrm{THz}$ has outstanding performance and will be used for flight. A chain covering 1.83 to $1.91 \mathrm{THz}$ meets the requirements, but only marginally. As a result, the final stage tripler has been re-tuned and is completing manufacturing. The best of the old or the new final stages will be delivered to HIFI. The multiplier chain covering $1.57-1.7 \mathrm{THz}$ has been unexpectedly difficult, due to a number of device layout and processing problems. As a result, it may be necessary to resort to a x18 approach for this band, starting from about $88 \mathrm{GHz}$. The implementation approach is identical to the band 5 approach with an adjustable structure, except the lengths have been adjusted to compensate for slightly different feed horn locations and chain lengths. Figure 12 shows the frequency and power performance to data of this effort.

\section{HIFI Band 6 LO Measured Results}

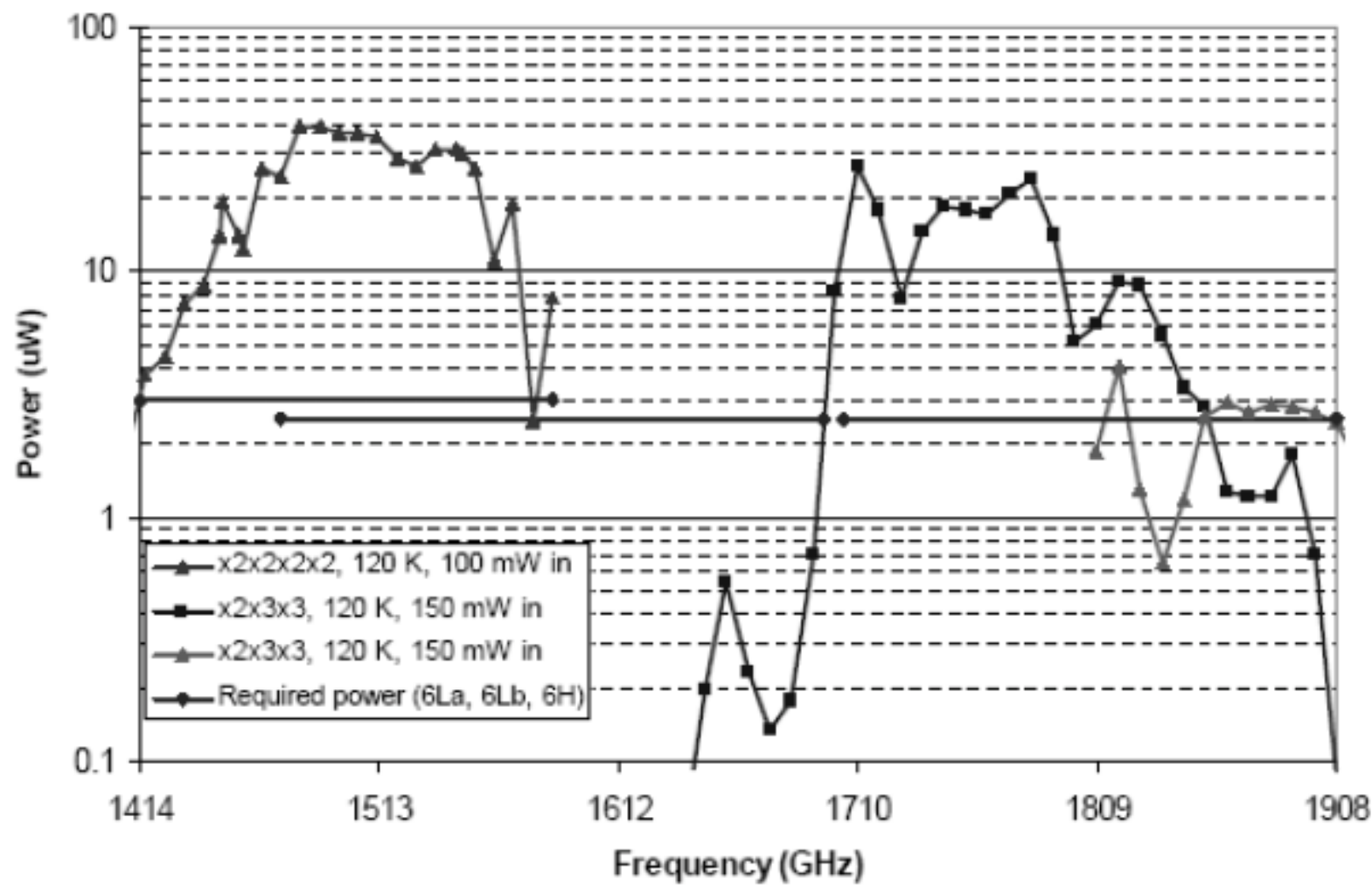

Figure 12. Summary table of results in the 1.41 to $1.91 \mathrm{THz}$ region. This will be covered with 4 multiplier chains. The results are the composite of the three working chains.

\section{SYSTEM ENGINEERING}

A number of operational details still need to be addressed for the use of the high frequency components. The first open question is the noise of an amplifier driven multiplier chain. The amplifiers are operated saturated, which should reduce AM noise at the expense of FM noise in the form of harmonics and intermodulation products. Since the amplifiers drive varactor multipliers, harmonics should not be an issue, but the intermodulation products could be a serious problem if the input signal does not have high spectral purity. The major unknown is the efficiency in AM to FM and FM to AM conversion in the multiplier chains. In general, the better behaved the output power and bias are, the less of a problem this should be. Unfortunately there is no easy way to calculate or measure this at the multiplier level. The 
other noise question is the optimal bias point for noise in a multiplier. To date, there has been no study of multiplier noise as a function of bias point and input power.

The power actually required by a phonon cooled HEB device remains a difficult question. It has been concluded that the isothermal method commonly used by designers of mixers substantially underestimates the local oscillator power requirement at the optical element of the mixer ${ }^{21}$. The final open question is the stability of the local oscillator in the HIFI operational mode, which reduces the input power to the multiplier chain. Unfortunately, the optimum bias point, where the output power is a maximum, is a function of input power. As a result, reducing the input power sometimes results in a condition where the output power does change with bias.

Operationally there are a number of challenges to overcome as well. The power amplifiers typically have about $3 \mathrm{~dB}$ of power variation over the band. As a result, an amplifier that barely achieves specification at one frequency can produce $3 \mathrm{~dB}$ more elsewhere in the band with the same bias conditions. Thus, a loss of frequency knowledge could be fatal for a multiplier. Additionally, acceptable safe bias and input power ranges for multipliers are a strong function of frequency. As a result, there is no one set of bias and power levels that are safe and produce the desired output power across the entire band for the higher frequency multipliers. This is further complicated by a bias supply that is designed to handle different multipliers with different bias requirements. The result is an operational challenge to determine how to assure that the L.O. system is operated in a "safe" manner, without destroying itself.

\section{CONCLUSIONS}

The technology development for the high frequency components of Herschel HIFI is nearly complete. At this time, the HIFI band 5 and 6 mixers are in the final phases of the flight qualification program, the flight build is nearly complete and the flight test is underway. The custom built devices, like diodes and high electron mobility transistors, have all been fully qualified and delivered to the HIFI instrument. The power amplifiers and isolators have all been qualified, manufactured, flight accepted and delivered to the HIFI instrument, with performance meeting or exceeding expectations. The multiplier chains for $1120-1250 \mathrm{GHz}$ have been qualified, manufactured and flight accepted with delivery expected early in the summer of 2004. The performance of these LO elements exceed all expectations. The band 6 local oscillators for 1410-1910 GHz are progressing well with a few chains ready for final flight integration and test. Delivery of these chains appears on track for the end of 2004. Should the few small system level questions be resolved successfully, HIFI will be an unprecedented instrument in capability, once the Herschel Space Observatory is launched.

\section{ACKNOWLEDGEMENT}

This research was carried out at the Jet Propulsion Laboratory, California Institute of Technology, under a contract with the National Aeronautics and Space Administration.

\section{REFERENCES}

1. M.W. de Graauw, N.D. Whyborn, E. Caux, T.G. Phillips, J. Stutzki, \& K. Wafelbakker, "The Herschel- HIFI instrument," Proc. SPIE vol. 5498, in Press, 2004.

2. J.C. Pearson, I. Mehdi, E. Schlecht, F. Maiwald, A. Maestrini, J. Gill, S. Martin, D. Pukala, J. Ward, J. Kawamura, W.R. McGrath, W.A. Hatch, D. Harding, H.G. Leduc, J.A. Stern, B. Bumble, L. Samoska, T. Gaier, R. Ferber D. Miller, A. Karpov, J. Zmuidzinas, T. Phillips, N. Erickson, J. Swift, Y.-H. Chung R. Lai, and H. Wang, 2002, “THz Frequency Receiver Instrumentation for Herschel's Heterodyne Instrument for Far Infrared (HIFI)," Proc. SPIE vol. 4850, 650, 2002.

3. I. Mehdi, "THz local oscillator technology," Proc. SPIE vol. 5498, in Press, 2004.

4. A. Karpov, D. Miller, J.A. Stern, B. Bumble, H.G. Leduc, \& J. Zmuidzinas, "Low-noise SIS far infrared heterodyne receiver," Proc. SPIE vol. 5498, in Press, 2004.

5. T. Klien, M. Cienanowicz, R. Guesten, C. Kasemann, T. Kirst, S. Philipp, \& A. Wunsch, "The local oscillator system for Herschel/HIFI," Proc. SPIE vol. 5498, in Press, 2004. 
6. R.T. Schieder, O. Siebertz, F. Schloeder, C. Gal, J. Stutzki, \& V. Natale, "Wideband spectrometer for HIFIFIRST," Proc. SPIE vol. 4013, 313, 2000.

7. R.T. Schieder, "Large bandwidth spectrometers for heterodyne receivers," Proc. SPIE vol. 5498, in Press, 2004.

8. L. Ravera, Ph. Cais, M. Giard, G. Montignac, J.L. Noullet, E. Caux, J.M. Debats, J.B. Begueret, D. Navarro, \& N. Laigne, "Wideband high-resolution versatile spectrometer proposed for FIRST-HIFI," Proc. SPIE vol. 4013, 305, 2000.

9. M. Belgacem, L. Ravera, E. Caux, P. Cais, \& A. Cros, "The high resolution versatile digital spectrometer of HIFIHSO,” New Astronomy 9, 43, 2004.

10. A. Karpov, D. Miller, F. Rice, J. Zmuidzinas, J.A. Stern, B. Bumble, \& H.G. Leduc, "Low noise SIS mixer for the band 1.1 to $1.25 \mathrm{THz}$ of the Herschel space radiotelescope," Proc. 14 ${ }^{\text {th }}$ Int. Symp. Space THz Tech., in Press 2003.

11. A. Karpov, D. Miller, F. Rice, J. Zmuidzinas, J.A. Stern, B. Bumble, \& H.G. Leduc, "Low noise $1.2 \mathrm{THz}$ SIS mixer," Proc. $15^{\text {th }}$ Int. Symp. Space THz Tech., in Press 2004.

12. H.F. Merkel, "Hot electron bolometer receivers for Herschel and beyond," Proc. SPIE vol. 5498, in Press, 2004.

13. S. Cherednichenko, J.W. Kooi, T. Berg, V. Drakinskiy, \& E. Kollberg, "Stability investigation of hot-electron bolometer mixers," Proc. SPIE vol. 5498, in Press, 2004.

14. J.C. Pearson, R. Guesten, T. Klein, \& N.D. Whyborn, "Local oscillator system for the heterodyne instrument on FIRST (HIFI)," Proc. SPIE vol. 4013, 264, 2000.

15. R.R. Ferber, T.C. Gaier, J.C. Pearson, L.A. Samoska, M. Wells, G. Swift, P. Yocom, Y. Chung \& A. Campbell, "W-band power amplifier development for the Herschel HIFI instrument," Proc. SPIE vol. 4855, 468, 2002.

16. N.R. Erickson, "Very low loss wideband isolators for mm-wavelengths," IEEE MTT-S Digest vol. 2, 1175, 2001.

17. S. Martin, B. Nakamura, A. Fung, P. Smith, J. Bruston, A. Maestrini, F. Maiwald, P. Siegel, E. Schlecht, \& I. Mehdi, "Fabrication of 200 to $2700 \mathrm{GHz}$ multiplier devices using GaAs and metal membranes," IEEE MTT-S Digest vol 3, 1641, 2001.

18. F. Maiwald, E. Schlecht, A. Maestrini, G. Chattopadhyay, J.C. Pearson, D. Pukala, \& I. Mehdi, "Terahertz frequency multiplier chains based on planar Schottky diodes," Proc. SPIE vol. 4855, 447, 2002.

19. J. Ward, F. Maiwald, A. Maestrini, G. Chattopadhyay, E. Schlecht, J. Gill, \& I. Mehdi, "1400-1900 GHz local oscillators for the Herschel Space Observatory," Proc. 14 $4^{\text {th }}$ Int. Symp. Space THz Tech., in press 2003.

20. J. Ward, G. Chattopadhyay, A. Maestrini, E. Schlecht, J. Gill, F. Maiwald, \& I. Mehdi, "Tunable all-solid-state local oscillators to $1900 \mathrm{GHz}$," Proc. $15^{\text {th }}$ Int. Symp. Space THz Tech., in press 2004.

21. C.-Y.E. Tong, D. Meledin, D. Loudkov, R. Blundell, N. Erickson, J. Kawamura, I. Mehdi \& G. Gol'tsman, “A 1.5 THz hot-electron bolometer mixer operated by a planar diode based local oscillator," IEEE MTT-S Digest vol. 2, $751,2003$. 